\title{
Reoperation for prosthesis dehiscence caused by aortitis
}

\author{
Hiroshi Tanaka, MD, Hitoshi Ogino, MD, Hitoshi Matsuda, MD, and Hiroaki Sasaki, MD, Osaka, Japan
}

Aortitis is a disease that affects the aortic valve and results in aortic insufficiency, requiring prosthesis implantation. Consequently, dehiscence of the implanted prosthesis may occur after the operation. In this situation, re-do aortic root replacement is necessary in most cases, but transannular stitches cannot be used to secure the aortic-ventricular continuity because of the destroyed annulus. We performed 3 cases of re-do aortic root replacement by reconstructing the left ventricular outflow tract (LVOT) with a mechanical valve conduit and obtained good results.

\section{CLINICAL SUMMARY}

After establishing cardiopulmonary bypass and clamping the ascending aorta, the aortic root or root prosthesis is dissected down cylindrically to the aortic-ventricular junction, creating the buttons of the coronary artery ostia. After making a "stump" on the LVOT, mattress sutures are placed from outside of the stump to inside on the fibrous and muscular portions, and reinforced around the stump with a "belt-like" Teflon felt, as originally performed by Ando and colleagues ${ }^{1}$ (Figure $1, A$ ). If the membranous septum

From the Department of Cardiovascular Surgery, National Cerebral and Cardiovascular Center, Suita, Osaka, Japan.

Disclosures: Authors have nothing to disclose with regard to commercial support. Received for publication Jan 11, 2011; accepted for publication March 21, 2011; available ahead of print April 19, 2011.

Address for reprints: Hiroshi Tanaka, MD, Department of Cardiovascular Surgery, National Cerebral and Cardiovascular Center, 565-8565, 5-7-1, Fujishirodai, Suita, Osaka, Japan (E-mail: hirtanak@hsp.ncvc.go.jp).

J Thorac Cardiovasc Surg 2011;142:1274-5

$0022-5223 / \$ 36.00$

Copyright (c) 2011 by The American Association for Thoracic Surgery doi:10.1016/j.jtcvs.2011.03.016 is friable, the sutures are placed below it. In this case, atrioventricular block will occur at a high rate postoperatively. ${ }^{4}$ We use a handmade composite graft with a small length of "skirt" (Figure 1,B) to reconstruct the LVOT. The coronary artery is reattached to the graft as a cuff with Teflon felt reinforcement. The graft interpose technique is used if the coronary cuff is friable to avoid mechanical stress on the anastomosis.

\section{CASE 1}

A 39-year-old man with severe aortic insufficiency and aortic root aneurysm due to Behçet's disease underwent aortic root replacement with conventional transannular stitches. One year after the operation, cardiac failure developed in the patient and echocardiography showed a pseudoaneurysm of the aortic root compressing the left coronary artery. He underwent re-do aortic root replacement with a mechanical valve conduit. Pathologic findings of the resected aortic wall showed active aortitis with no signs of infection. Four years after the operation, computed tomography (CT) showed no pseudoaneurysm, but the patient did have multiple right coronary artery stenosis due to Behçet's disease despite corticosteroid therapy.

\section{CASE 2}

A 50-year-old man with severe aortic regurgitation due to polyarteritis nodosa, which had been treated with oral corticosteroids, underwent aortic valve replacement. Three months after the operation, he underwent aortic root replacement with a composite valve graft for a pseudoaneurysm without infection. One year after the second operation,

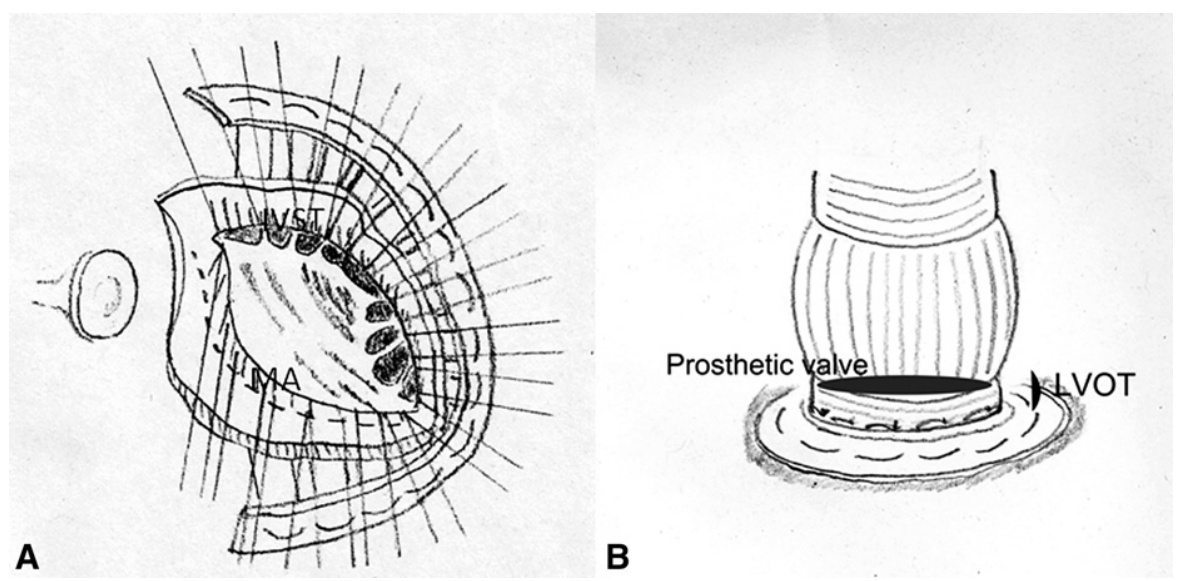

FIGURE 1. A, Mattress sutures on the fibrous muscular portions of the LVOT reinforced around the stump with a "belt-like" Teflon felt. B, Hand-made composite graft with a small length of "skirt" using a Valsalva graft (Vascutek, Renfrewshire, Scotland). MA, Mitral annulus; IVST, interventricular septum; LVOT, left ventricular outflow tract. 


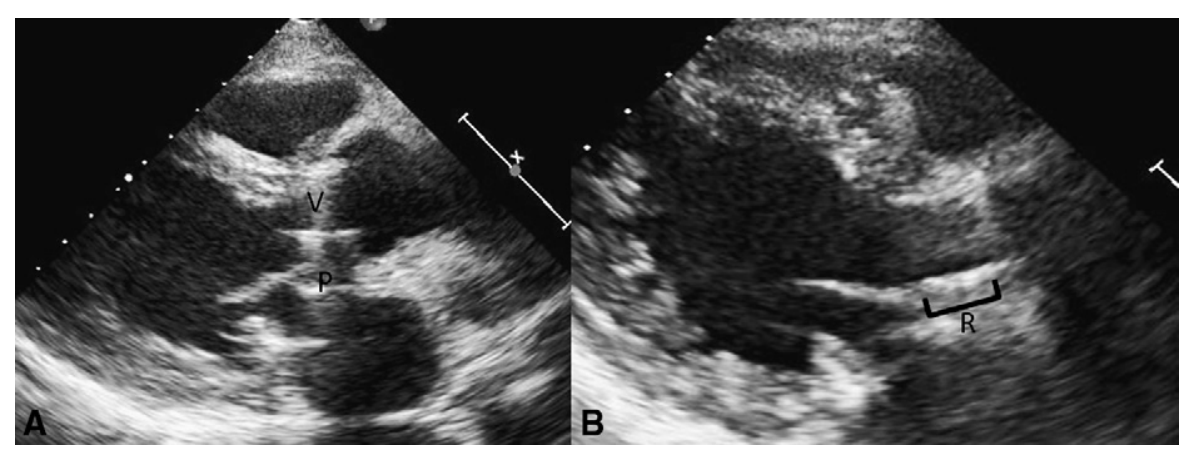

FIGURE 2. A, Preoperative echocardiography shows the detached prosthetic aortic valve and pseudoaneurysm on the LVOT. B, Postoperative echocardiography shows the reconstructed LVOT with a vascular prosthesis. $V$, Detached prosthetic valve; $P$, pseudoaneurysm on the LVOT; $R$, reconstructed LVOT.

chest CT showed multiple pseudoaneurysms, and the patient was referred to the National Cerebral and Cardiovascular Center. He underwent re-do aortic root replacement with a mechanical valve conduit. Pathologic findings of the resected aortic wall showed aortitis with no signs of infection. CT showed no pseudoaneurysm 3 years after the operation.

\section{CASE 3}

A 49-year-old woman with severe aortic regurgitation and mild mitral regurgitation underwent aortic valve replacement and mitral annuloplasty. Three months after the operation, echocardiography showed dehiscence of the aortic prosthesis. She underwent re-do aortic valve replacement; however, 6 months after the operation, echocardiography showed a pseudoaneurysm on the LVOT and dehiscence of the prosthesis (Figure 2,A). She underwent re-do aortic root replacement with a mechanical valve conduit. She required a pacemaker implantation because of postoperative atrioventricular block. Pathologic findings of the resected aortic wall showed giant cell aortitis. Two years after the operation, echocardiography showed no signs of pseudoaneurysm or dehiscence of the prosthesis (Figure 2, B).

\section{DISCUSSION}

Dehiscence of the prosthesis after valve or root replacement for aortitis has been reported, and the results of reoperation for this condition are poor. Patients with Behçet's disease have a high rate of dehiscence, even though they receive corticosteroid therapy. ${ }^{1}$ We use the LVOT to anchor the vascular prosthesis: the muscular and fibrous portions. Aortitis rarely affects the myocardium and intracardiac fibrous portion ${ }^{2}$; therefore, this technique would secure the long-term outcome of the procedure. Physiologically, the "belt-like" Teflon felt reinforces the anastomosis and equally distributes the mechanical stress circumferentially. ${ }^{1}$ The "skirt" technique enables a mechanical valve to be used in middle-aged patients, although a stentless bioprosthesis and homograft, which are more pliable than a sawing ring of prosthetic valve but might require reoperation because of structural deterioration, are preferred to reduce the stress on the anastomosis. ${ }^{3}$ Despite the technical aspect, the most important clinical point to prevent a poor outcome is postoperative reduction of inflammation on the anastomoses with corticosteroid therapy.

\section{CONCLUSIONS}

This technique would be useful in re-do aortic root replacement even in patients with an active phase of aortitis.

\section{References}

1. Ando M, Kosakai Y, Okita Y, Nakano K, Kitamura S. Surgical treatment of Behçet's disease involving aortic regurgitation. Ann Thorac Surg. 1999;68: 2136-40.

2. Marzban M, Mandegar MH, Karimi A, et al. Cardiac and great vessel involvement in "Behcet's disease". J Card Surg. 2008;23:765-8.

3. Jeong DS, Kim KH, Kim JS, Ahn H. Long-term experience of surgical treatment for aortic regurgitation attributable to Behçet's disease. Ann Thorac Surg. 2009;87: 1775-82.

4. Krasopoulos G, David TE, Armstrong S. Custom-tailored valved conduit for complex aortic root disease. J Thorac Cardiovasc Surg. 2008;135:3-7. 